\title{
Wi-Fi For Indoor Device Free Passive Localization (DfPL): An Overview
}

\author{
R. Gunasagaran ${ }^{1}$, L.M. Kamarudin ${ }^{2}$, A. Zakaria ${ }^{3}$ \\ ${ }_{1,2,3}$ Centre of Excellence for Advanced Sensor Technology, University Malaysia Perlis, Malaysia \\ ${ }^{1,2}$ School of Computer and Communication Engineering, University Malaysia Perlis, Malaysia \\ ${ }^{3}$ School of Mechatronic Engineering, University Malaysia Perlis, Malaysia
}

\begin{tabular}{l} 
Article Info \\
\hline Article history: \\
Received Feb 23, 2019 \\
Revised Feb 14, 2020 \\
Accepted Mar 3, 2020 \\
\hline
\end{tabular}

\section{Keywords:}

device free passive localization internet of things

wi-fi

\begin{abstract}
The world is moving towards an interconnected and intercommunicable network of animate and inanimate objects with the emergence of Internet of Things (IoT) concept which is expected to have 50 billion connected devices by 2020 . The wireless communication enabled devices play a major role in the realization of IoT. In Malaysia, home and business Internet Service Providers (ISP) bundle Wi-Fi modems working in $2.4 \mathrm{GHz}$ Industrial, Scientific and Medical (ISM) radio band with their internet services. This makes Wi-Fi the most eligible protocol to serve as a local as well as internet data link for the IoT devices. Besides serving as a data link, human entity presence and location information in a multipath rich indoor environment can be harvested by monitoring and processing the changes in the Wi-Fi Radio Frequency (RF) signals. This paper comprehensively discusses the initiation and evolution of Wi-Fi based Indoor Device free Passive Localization (DfPL) since the concept was first introduced by Youssef et al. in 2007. Alongside the overview, future directions of DfPL in line with ongoing evolution of Wi-Fi based IoT devices are briefly discussed in this paper.
\end{abstract}

Copyright (c) 2019 Institute of Advanced Engineering and Science. All rights reserved.

\author{
Corresponding Author: \\ R. Gunasagaran, \\ Centre of Excellence for Advanced Sensor Technology, \\ University Malaysia Perlis, \\ Perlis, Malaysia. \\ Email: gunasagaran.rajeshkumar@gmail.com
}

\section{INTRODUCTION}

Human kind with the advancement of technology is striving towards intelligence in every corner of their lives from a simple light bulb to a complex smart home setup. Introduction of Internet of Things (IoT) concept became a major driver working towards the fruition of this strive. IoT is the concept of connecting animate and inanimate as well as living and non-living things via an interconnected and intercommunicable network to ease and automate the management of each connected entity [1]. The automation is realized by incorporating decision making process in the edges that collect the data, fogs that relay the data or the cloud. It is projected that IoT will see 50 billion connected devices by 2020 [2]. With communication being the major key point for the success of IoT and having multiple wired communications around being a hindrance to daily human activities, wireless communication enabled devices became the highlight and saw further advancement in terms of energy consumption, size and cost in this era of IoT.

In Malaysia, home and business Internet Service Providers (ISP) bundle Wi-Fi modems working in $2.4 \mathrm{GHz}$ Industrial, Scientific and Medical (ISM) radio band in their internet service packages. With the government's efforts to reduce the price and increase the speed of the internet service packages, the number of households and businesses with internet connections are expected to raise in the coming years [3,4]. Thus, we can foresee Wi-Fi modems being ubiquitously available indoors in Malaysia. This infrastructure supports seamless deployment of Wi-Fi enabled IoT devices and plays both the roles of local as well as internet data link. Humans spend over $80 \%$ of their time indoors either in their house, office or shopping areas [5]. It is 
known that human body consists of more than $70 \%$ water and $2.4 \mathrm{GHz}$ is the resonance frequency of water [6, 7]. This denotes human bodies can absorb Radio Frequency (RF) signals in the $2.4 \mathrm{GHz}$ frequency range [7]. Compare to outdoor environments, indoor environments are rich in multipath where a transmitted RF wireless signal can propagate along multiple paths with each signal having different amplitude and phase which are combined at the receiver to reproduce a distorted version of the original transmitted signal [7]. Reflection, diffraction, refraction and absorption of the RF signal along these multiple paths induce multipath fading which either increase or decrease the Received Signal Strength Indicator (RSSI) at the receiver [8, 9]. Besides the multipath fading caused by the indoor environment, human presence and mobility effects the RF signals as well due to the aforementioned property of the signals.

Outdoor localization is accurately executed with the help of Global Positioning System (GPS). The GPS device requires direct Line-of-Sight (LOS) communication with the GPS communication satellites orbiting at the Low Earth Orbit to retrieve the latitude and longitude information [10]. This requirement makes it not practical to use GPS indoors as the transmitted signal cannot penetrate obstacles such as walls and metals $[10,11]$. To cater indoor localization problem early works were RF based Device bound Active Localization (DbAL) systems whereby each localized entity is required to carry a Wi-Fi transceiver such as in RADAR [12] and Horus [13] or a RFID tag such as in LANDMARC [14]. However, attaching a device to an entity for indoor localization requires additional resources in terms of devices and professional labor in some cases. This in turn paved the way towards RF based Device free Passive Localization (DfPL) systems. Figure 1 shows the general difference between DbAL and DfPL.

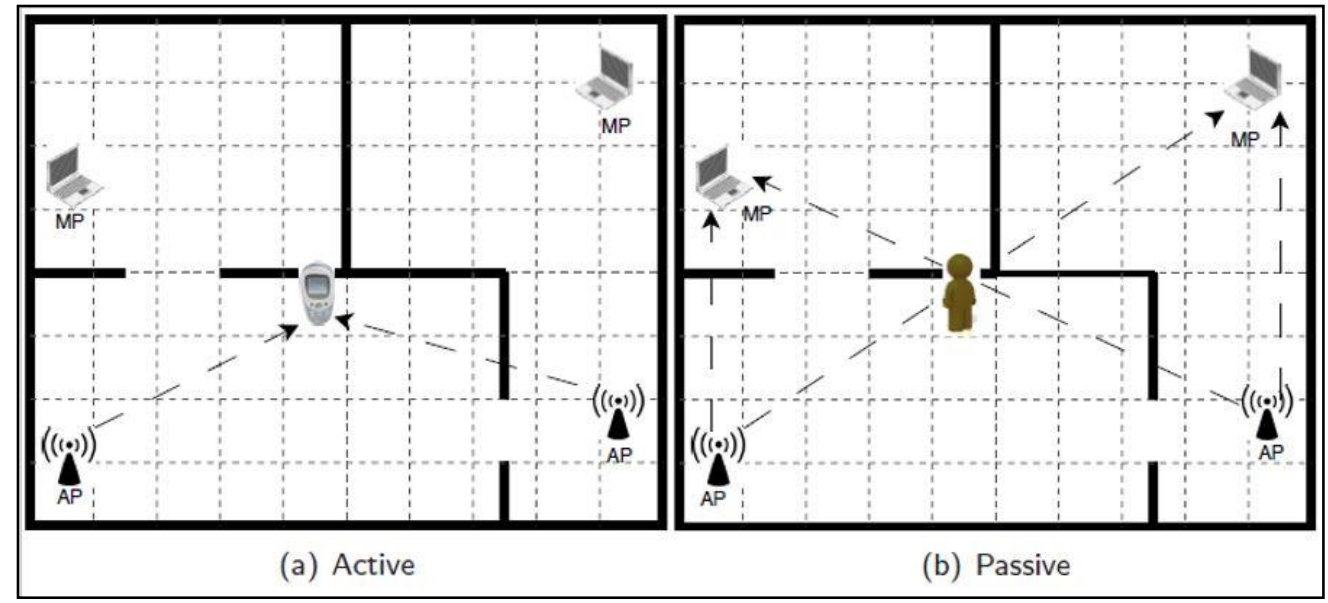

Figure 1. (a) Device bound Active Localization (DbAL) [15] (b) Device free Passive Localization (DfPL)

[15]

DfPL was first introduced by Youssef et al. in 2007. A DfPL system is envisioned as a system with the ability to detect, track and identify entities in an Area of Interest (AOI) without the entities carrying any specific devices nor actively participating in the localization process [8]. In concept, the system is expected to capture changes in the AOI by monitoring and processing variations in the received physical signals collected in one or more Monitoring Points (MP) [8]. Since the introduction, many DfPL systems were developed based on the pioneer 802.11 protocol Wi-Fi devices as well as other protocol devices. The early systems based on Wi-Fi devices were using RSSI variation as the data to detect, localize and track entities. Those systems came in an incremental manner with improvement compare to [8]. Among the improved systems were Nuzzer [9], RASID [16] and Ichnaea [17] to name a few. Alongside RSSI variation, with the application of Multiple-Input and Multiple-Output (MIMO) antenna technology in Wi-Fi devices and the development of software to extract Channel State Information (CSI) from commodity off the shelve Network Interface Cards (NIC), CSI variation came into the picture of DfPL as another extractable data from Wi-Fi to execute localization and tracking of entities $[5,18,19]$. Aside from using Wi-Fi RSSI and CSI, there are also some recent systems that use Wi-Fi probe requests from devices to cluster and localize entities [20, 21].

Besides the implementation on Wi-Fi infrastructure, DfPL was also experimented with RSSI of devices working on sub $1 \mathrm{GHz}$ wireless communications with a more denser deployment of transmitters and receivers $[22,23]$. Among the early improvements to these systems was the usage of camera for recalibration [24]. Some recent works on these systems focus on the reduction of the number of nodes while retaining the accuracy of the systems [25, 26]. Another variation of DfPL was implemented on RSSI of RFID infrastructure using monostatic or bistatic antennas connected to RFID readers that reads the deployed passive or active RFID tags using backscatter communication [27-29]. RFID based DfPL systems are among the early systems that 
proposed Artificial Neural Network (ANN) as well as posture and fall detection [30, 31]. Besides using RSSI, some other variants of RFID based systems were implemented using the coupling effect of passive RFID tags by placing the antennas of tags parallel and close to each other making one of the tags unreadable without an interference and using Angle of Arrival (AoA) [32, 33].

DfPL was also realized using ambiently available FM signals by collecting and processing the signal properties using Software Defined Radios (SDR) [34-36]. Region based activity monitoring was introduced in a concealed manner in FM based system [34]. Besides FM based systems, there are also cellular based systems that monitor and process GSM signals for entity detection [37]. There are also DfPL systems that use signal subspace eigenvector collected with receiver antenna arrays and Ultra-Wideband (UWB) [38, 39]. All the above-mentioned systems work on the principle of location-based scheme with most of them requiring a fingerprint for the detection, localization and tracking process [40].

After the introduction of Wireless Sensor Network (WSN), the principle of link-based scheme was introduced in the form of Radio Tomographic Imaging (RTI) [41-43]. RTI is implemented by deploying WSN nodes in high numbers to concentrate the AOI with node links and performing DfPL tasks by monitoring the fading and shadowing effects on the links [41]. The concept of RTI is also implemented in some RFID based DfPL systems [44, 45]. Alongside all the RF based systems mentioned, there are also systems using camera, infrared (IR) sensors, passive infrared (PIR) sensors, carbon dioxide (CO2) sensors, seismic sensors etc. to perform localization tasks in a passive manner. However, infrared and optical imaging systems fail in dark environments and presence of smoke [41]. On the other hand, $\mathrm{CO} 2$ and seismic systems can fail due to ambiently available $\mathrm{CO} 2$ and sounds. Thus, RF based systems are deemed to be superior as the RF signals can penetrate through obstacles such as smoke and wall [41].

Among the RF based systems, Wi-Fi based DfPL is the most promising as Wi-Fi modems are ubiquitously available in the households, work places, cafes, shopping mall etc. providing the required infrastructure for the deployment of Wi-Fi based IoT devices that can participate in the DfPL tasks and add more value to the infrastructure. The remainder of the paper is organized as follows. Section 2 discusses possible application of DfPL systems. Following that, Section 3 explores the initiation and evolution of Wi-Fi based DfPL systems. Section 4 reviews the characteristics of these systems. In Section 5, future direction is discussed and Section 6 concludes the paper.

\section{POSSIBLE APPLICATIONS OF DEVICE FREE PASSIVE LOCALIZATION (DFPL) SYSTEMS}

DfPL systems can be applied to shopping navigation, intruder detection, disaster rescue, energy management and assisted living. In terms of shopping navigation, customer location information can be harvested by a centralized server to provide location-based services and navigation throughout the available items in a shop or the available shops in a shopping mall via interactive panels or venue specific mobile applications. A similar system can also be adopted to libraries, museums etc. As imaging and IR system fail beyond the detection of intruders, DfPL systems can be deployed to detect, localize and further track an intruder in an AOI.

For disaster rescue, link-based DfPL systems can be rapidly deployed around the AOI to assist the rescue efforts by localizing the victims. In terms of energy management, DfPL systems can be used to intelligently manage home as well as office appliances for heating, ventilation and air conditioning based on the presence of a human to reduce waste of energy. For assisted living of elderly and impaired people, DfPL systems can be used to localize and monitor their daily activities based on locations to alert their emergency contact in the presence of abnormalities in their daily activities.

\section{EVOLUTION OF WI-FI BASED DEVICE FREE PASSIVE LOCALIZATION (DFPL) SYSTEMS}

The ground work for Wi-Fi based DfPL began in the form of DbAL. In the year 2000, RADAR [12] was the earliest work on localization using 802.11 protocol to provide location aware services via triangulation where distance of devices were calculated as a function of RSSI. This work used RSSI of UDP packets broadcasted by laptop mobile stations from pre-determined locations collected at a computer base station as profile data to determine the location of devices using empirical method of nearest neighbor and signal propagation modelling. Following that the work in [7] used similar device configuration as [12] and used probability distribution-based method for localization to address noisy channel problem and introduced radio map to simplify the localization algorithm. [46] implemented active scanning at laptop mobile stations to collect Access Point (AP) probe RSSI data used for device location determination process and was among the early work that discussed temporal and spatial variation. Temporal variation is the property of RSSI at a same location that changes over time due to the alteration in the physical environment [46]. On the other hand, spatial variation is divided into large-scale variation and small-scale variation. Large-scale variation is the changes in 
RSSI over distance due to attenuation [46]. Small-scale variation is the changes in the RSSI over small distance in order of wavelength about 12.5 centimeters for $2.4 \mathrm{GHz}$ frequency range [46]. Horus [13] proposed using Gaussian distribution to address temporal variation in the device localization process and compensator modules to address spatial variation. These are among the early works that correlated RSSI RF link quality indicator to location and paved the way towards the horizon of DfPL.

In the year 2007, DfPL concept was first introduced in [8] where the system architecture used APs in the same manner as [46] but switched the laptop mobile station to static MPs and localize human entities instead of devices. [8] utilized moving average and moving variance for human entity detection. As adapting physical signal propagation models in indoor RF problems with heavy multipath fading was very difficult, DfPL systems utilized fingerprints in terms of passive radio maps introduced in [8] for the localization tasks [30]. A deterministic radio map represents each location with the RSSI received from each AP by a single value such as mean, variance or standard deviation, while a probabilistic radio map represents each location with a distribution of RSSI received from each AP [47]. As the performance of detection method in [8] degrades in real environment, [48] proposed and evaluated maximum likelihood estimator for detection. [49] evaluated deterministic and probabilistic algorithms for DfPL and proved Histogram based non-parametric probabilistic approach outperformed the other approaches. [50] deployed the DfPL system in large scale environment and introduced discrete space estimator using median with Euclidean distance, continuous space estimator using spatial averaging and time averaging to improve the performance of the system. Besides that, [50] also utilized relative variance to detect human entity at LOS. While the previous DfPL systems focus on localizing entities in an office environment, [51] narrow down the AOI to a corridor and implemented Kalman filter to improve tracking of the entity.

As collection of RSSI values to create a radio map is a labor intensive process, [47] proposed modelling RF propagation and human shadow effect using Ray Tracing and Uniform Theory of Diffraction (UTD) to automatically generate a passive radio map and further localize using Parzen Window based classifier with Gaussian smoothing kernel. RASID [16] suggested human presence detection as well as region segmentation using statistical anomaly detection besides introducing periodic calibration. [52] presented the usage of miniature embedded transceiver modules, background subtraction as well as algorithms such as $\mathrm{K}$ Nearest Neighbor (kNN), Multi-Layer Perceptron (MLP) Neural Network (NN) and Support Vector Machine (SVM) for localization. AROMA [15] proposed a similar method as [47] for radio map generation. Nuzzer [9] is an improved version of [50]. Pilot [18] opened another path forward for the Wi-Fi based DfPL systems in terms of CSI as an alternative to RSSI. CSI is a fine-grained physical layer information in units of amplitude and phase that describes channel property of RF links in subcarrier level for an Orthogonal Frequency Division Multiplexing (OFDM) based system [18]. Ichnaea [17] using RSSI proposed tracking using Particle filter.

[53] utilized CSI amplitude as feature to detect human entity in a near circular region at the receiver and determine the direction angle of the human entity in the region. [54] using RSSI proposed detection of occupancy by estimating the number of people utilizing available Wi-Fi infrastructure. [19] introduced Ks factor to estimate the receiver sensitivity and by doing so suggested better placement for the receiver to increase the sensitivity and reduce the dead spots in CSI based systems. [55] introduced Single Board Computer (SBC) Raspberry Pi with Wi-Fi module as a MP and proposed a method that transfers the RSSI model used to locate a human entity obtained at a source environment to the end user environment. [56] evaluated single feature Probabilistic Neural Network (PNN) with RSSI as well as multi feature PNN with features extracted from RSSI and proved multi feature PNN to be superior. MaTrack [57] utilized CSI phase to identify moving target's angle information using proposed Dynamic MUSIC algorithm. DfPL MPs can use active scanning to get link quality information from the AP probes. As the opposite is possible by using sniffers, [20] used Wi-Fi enabled device probe requests to detect and cluster occupancy.

[58] used clustering to detect coarse grained region-based localization without intensive site survey utilizing CSI. [59] proposed a RSSI based walking speed measurement method for DfPL. For CSI based DfPL systems, [60] suggested using DBSCAN to reduce the noise and Principle Component Analysis (PCA) to reduce the high dimensionality of the fingerprint. [61] proposed an auto calibration method for CSI based systems. [5] suggested localization using Bayes Classification as well as evaluated Kalman filter and Bayesian filter for tracking proving Kalman filter to be superior. [21] is similar to [20] in concept. The initiation and evolution of Wi-Fi based DfPL systems strongly prove ubiquitously available Wi-Fi infrastructure is highly capable in supporting detection, localization and tracking of human entities.

\section{CHARACTERISTICS OF WI-FI BASED DEVICE FREE PASSIVE LOCALIZATION (DFPL) SYSTEMS}

This section discusses the characteristics of Wi-Fi based DfPL systems in terms of architecture, RF link quality data, localization methods, evaluation metrics, number of APs, MPs and size of AOI. 


\subsection{Architecture}

The general architecture of a Wi-Fi based DfPL system consist of 3 subparts which are the APs, MPs and application server. APs are commercial units or ad hoc connections created by devices. MPs on the other hand are computers or laptops equipped with a NIC, mobile phones or Wi-Fi enabled embedded devices. Application server is a centralized local or cloud computer that collects the data from the MPs to perform detection, localization or tracking tasks of human entities. There are several schemes for localization such as spot-based, cell-based and region-based as shown in Figure 2. For spot-based scheme, the training data is

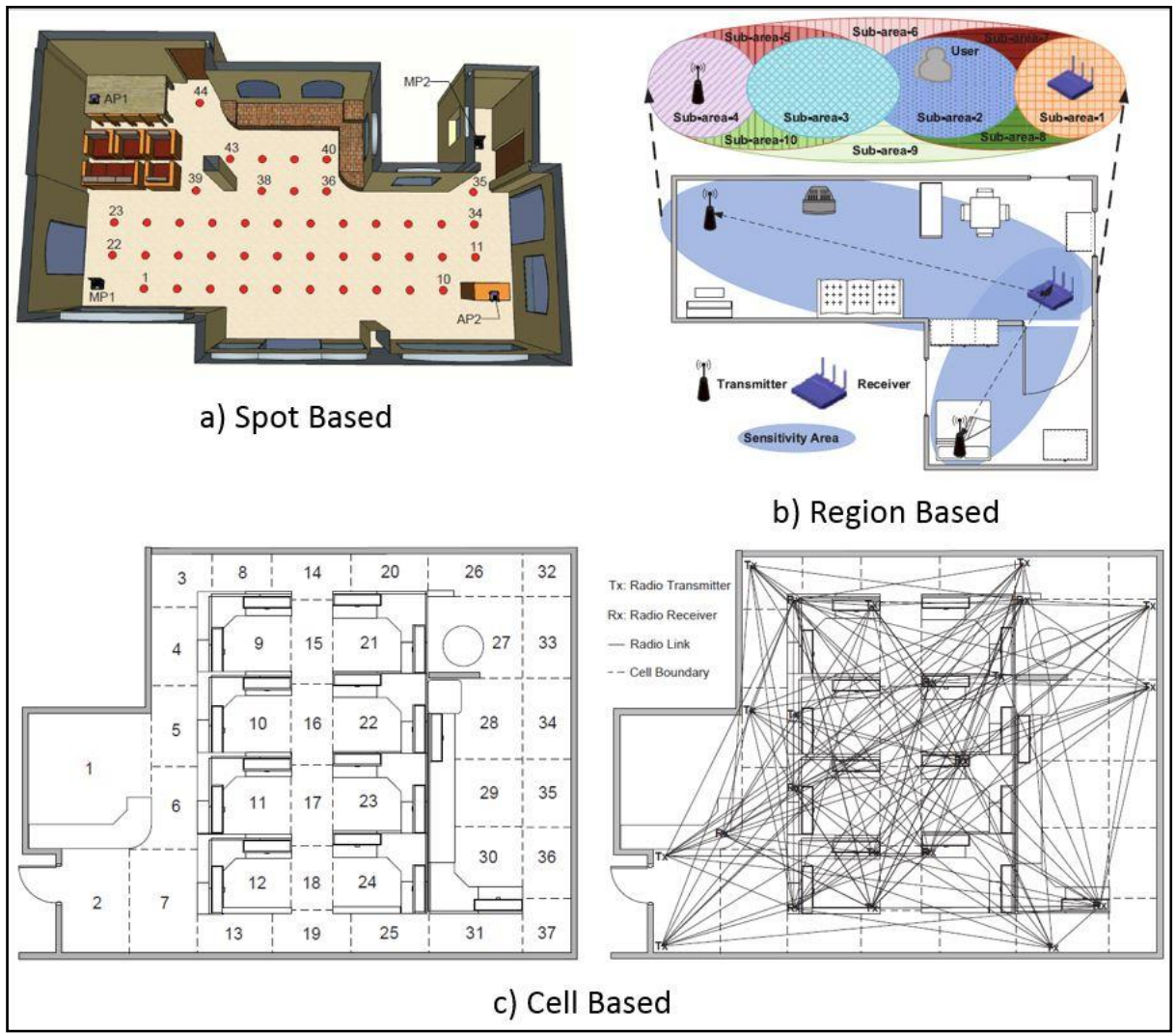

Figure 2. (a) Spot-Based [15] (b) Region-Based [58] (c) Cell-Based [23]

collected at defined spots and the localization task is done in a relative manner. On the other hand, cell-based scheme training data is collected in each cell with a degree of moving freedom to the human entity within the cell and the localization task is done in a discrete manner. Region- based scheme is a more coarse-grained scheme where localization is done based on separated big regions. Another common practice is the offline and online phase of the system. Offline phase is when the deterministic or probabilistic passive radio map of the AOI is manually created via data collection or automatically generated via modelling. While, the online phase is the real-time data processing phase that uses designated algorithms to perform localization tasks.

\subsection{Radio Frequency (RF) Link Quality Data}

RF link quality data that are commonly used in Wi-Fi based DfPL systems are RSSI and CSI. RSSI is more commonly extractable from all Wi-Fi enabled devices. CSI on the other hand is more fine-grained but can only be extracted from computers or laptops equipped with the latest generation NICs using specific software.

\subsection{Localization Methods}

Wi-Fi based DfPL systems utilized deterministic, statistical, probabilistic and training based methods individually or collectively to perform localization tasks. Among the deterministic methods implemented were moving average, moving variance, nearest neighbor, relative variance, mean, variance, standard deviation and maximum likelihood estimator. On the other hand, statistical method used was anomaly detection. Among the probabilistic methods employed were Gaussian based and Histogram based. Rather than that, training based methods used were MLP NN, SVM, PNN, Parzen Window based classifier and Bayes classifier.

\subsection{Evaluation Metrics}

Wi-Fi For Indoor Device Free Passive Localization (DfPL): An Overview (R. Gunasagaran et al) 
Among the initial evaluation metrics used for the systems were average distance error, median distance error and probability of error. These were measured from the distribution based on the difference in the actual and estimated location of a human entity in an AOI. Current systems use Precision, Recall and F1 Score as evaluation metrics. Precision evaluates the exactness and defined by the number of correct events detected divided by the number of total events detected [48]. Recall evaluates the completeness and defined by the number of correct events detected over the actual number of events in the experiment [48]. F1 score is the weighted average of precision and recall.

\subsection{Number of Access Points (AP), Monitoring Points (MP) and Size of the Area of Interest (AOI)}

The number of APs, MPs, size of the AOI and link quality indicator used by the Wi-Fi based DfPL systems discussed in this paper are summarized chronologically in Table 1. Some systems have AOIs with multiple sizes as indicated.

Table 1. Summary of Wi-Fi based DfPL systems

\begin{tabular}{|c|c|c|c|c|}
\hline DfPL System & Number of APs & Number of MPs & $\begin{array}{c}\text { Size of the AOI } \\
\text { (meter) }\end{array}$ & $\begin{array}{l}\text { Link Quality } \\
\text { Indicator }\end{array}$ \\
\hline [8] & 2 & 2 & - & RSSI \\
\hline [48] & 2 & 2 & $5.0 \mathrm{~m}$ by $5.0 \mathrm{~m}$ & RSSI \\
\hline [49] & 2 & 2 & $3.6 \mathrm{~m}$ by $5.0 \mathrm{~m}$ & RSSI \\
\hline [50] & 3 & 2 & $55.7 \mathrm{~m}$ by $38.8 \mathrm{~m}$ & RSSI \\
\hline [51] & 4 & 4 & $16.5 \mathrm{~m}$ & RSSI \\
\hline [47] & 2 & 2 & 65 square $\mathrm{m}$ & RSSI \\
\hline \multirow[t]{2}{*}{ [16] } & 4 & 3 & 1: 185 square $m$ & RSSI \\
\hline & 4 & 3 & 2: 139 square $m$ & \\
\hline [52] & 5 & 6 & $8.0 \mathrm{~m}$ by $8.0 \mathrm{~m}$ & RSSI \\
\hline [15] & 2 & 2 & 66 square m & RSSI \\
\hline [9] & 3 & 2 & 139 square m & RSSI \\
\hline \multirow[t]{2}{*}{ [18] } & 1 & 3 & $1: 7.0 \mathrm{~m}$ by $11.0 \mathrm{~m}$ & CSI \\
\hline & 2 & 2 & 2: 776 square $\mathrm{m}$ & \\
\hline \multirow[t]{3}{*}{ [17] } & 4 & 3 & 1: 186 square $m$ & RSSI \\
\hline & 7 & 2 & 2: 352 square $m$ & \\
\hline & 4 & 3 & 3: 140 square $m$ & \\
\hline [53] & 1 & 1 & - & CSI \\
\hline [54] & 2 & - & $9.0 \mathrm{~m}$ by $15.0 \mathrm{~m}$ & RSSI \\
\hline \multirow{2}{*}{ [19] } & 1 & 1 & $1: 12.7 \mathrm{~m}$ by $7.2 \mathrm{~m}$ & CSI \\
\hline & 1 & 1 & 2: 20 square $\mathrm{m}$ & \\
\hline [55] & 1 & 1 & - & RSSI \\
\hline [56] & 5 & 3 & - & RSSI \\
\hline \multirow[t]{3}{*}{ [57] } & 2 & 2 & $1: 7.0 \mathrm{~m}$ by $7.4 \mathrm{~m}$ & CSI \\
\hline & 2 & 2 & $2: 4.2 \mathrm{~m}$ by $4.5 \mathrm{~m}$ & \\
\hline & 2 & 4 & 3: 68 square m & \\
\hline \multirow[t]{2}{*}{ [58] } & 1 & 1 & $1: 18.4 \mathrm{~m}$ by $14.4 \mathrm{~m}$ & CSI \\
\hline & 1 & 1 & 2: 40 square m & \\
\hline [59] & 1 & 1 & $4.15 \mathrm{~m}$ & RSSI \\
\hline [60] & 2 & 2 & $6.0 \mathrm{~m}$ by $7.0 \mathrm{~m}$ & CSI \\
\hline [61] & 1 & 1 & - & CSI \\
\hline \multirow[t]{4}{*}{ [5] } & 4 & 3 & $1: 1.8 \mathrm{~m}$ by $12.0 \mathrm{~m}$ & CSI \\
\hline & 4 & 3 & $2: 8.5 \mathrm{~m}$ by $8.0 \mathrm{~m}$ & \\
\hline & 4 & 3 & $3: 9.0 \mathrm{~m}$ by $14.0 \mathrm{~m}$ & \\
\hline & 4 & 3 & 4: $11.6 \mathrm{~m}$ by $7.2 \mathrm{~m}$ & \\
\hline
\end{tabular}

\section{FUTURE DIRECTION}

Future path forward for Wi-Fi based indoor DfPL is tightly connected to the mainstream adaptation of Wi-Fi based IoT devices. Considering home and workplace are the two indoor environments humans spend most of their time on a daily basis, the key elements in the adaptation process are the fourth industrial revolution, smart home as well as the technological innovation which are fractions of the IoT concept. The fourth industrial revolution calls for the digitalization of industries via Artificial Intelligence (AI) equipped connected devices to automate processes. The digitalization efforts are steered towards the next stage of the industrial revolution which calls for man and machine cooperation. To achieve these industrial revolution visions, communication plays a major role with Wi-Fi as a practical candidate of choice. Wi-Fi based DfPL can clearly assist in the man and machine cooperation scenario where detection, localization and tracking of human entity becomes a fundamental requirement.

Smart home in general refers to home automation. Smart home systems and devices provide comfort and convenience to their owners by allowing control of the devices in a remote manner based on their preferences. To achieve this, the devices need to have a connection with internet access whereby ubiquitously available Wi-Fi infrastructure comes into the picture as a primary candidate. Besides that, these devices also 
promise to provide energy efficiency to their owners. DfPL can be a good companion for the smart home devices to cater energy efficiency by detecting the human presence. In terms of manufacturing process of the devices this can reduce cost and size by discarding unnecessary hardware purposely included to detect human presence. For the consumers, this reduces price of the devices. In terms of sustainability, this can reduce waste of valuable resources.

In terms of technological innovation, with the dawn of IoT era, more Wi-Fi enabled miniature energy efficient low-cost devices such as microcontrollers have emerged in the commercial market. Among those, ESP8266 and ESP32 to name a few. ESP32 in particular is proven capable of working in a long-range Wi-Fi communication of a typical 10 kilometer range when combined with a directional antenna on top of the conventional Wi-Fi range [62]. Besides that, there are also devices that bridge long-range communication to short-range Wi-Fi communication such as Lorafi [63]. These Wi-Fi enabled devices can be easily attached to simple and complex appliances with or without modifications based on individual case to make the appliances become a part of the interconnected IoT network where local as well as online monitoring and control is possible. Wi-Fi connections for these devices can be established seamlessly as the infrastructure is ubiquitously available.

As the world is moving towards the IoT ecosystem, DfPL can be implemented by having the Wi-Fi enabled IoT devices collect and send link quality information to a centralized local or online server periodically. With the technological advancement such as Edge AI, we can even see Wi-Fi enabled IoT edge devices performing basic human entity localization as they connect to the APs in the near future [64].

\section{CONCLUSION}

In the world intertwined with IoT network, Wi-Fi will be the major used protocol as the infrastructure is ubiquitously available in households, work places, shopping malls etc. This paper discussed the initiation and evolution of Wi-Fi based DfPL systems in line with this projection. Besides, the evolution of the systems clearly show they are capable in detection, localization and tracking of human entities. Thus, it can be concluded that Wi-Fi based DfPL systems in line with the development of IoT will be more successful in terms of adaptation compared to other DfPL systems that require additional hardware to be installed on top of the available infrastructures.

\section{ACKNOWLEDGMENT}

This work was supported in part by the Malaysian Technical University Network (MTUN), Grant No. 9002-00094/ 9028-00002.

\section{REFERENCES}

[1] R. Gunasagaran et al., "Internet of things: Sensor to sensor communication," in 2015 IEEE SENSORS, 2015, pp. 14.

[2] D. Evans, "The Internet of Things: How the Next Evolution of the Internet is Changing Everything," 2011.

[3] "Gobind urges telcos to provide cheaper broadband," The Star Online, 13-Jul-2018.

[4] B. K. Sidhu, "Internet access for all, vows Gobind," The Star Online, 04-Aug-2018.

[5] S. Shi, S. Sigg, L. Chen, and Y. Ji, "Accurate Location Tracking From CSI-Based Passive Device-Free Probabilistic Fingerprinting," IEEE Trans. Veh. Technol., vol. 67, no. 6, pp. 5217-5230, Jun. 2018.

[6] G. Deak, K. Curran, J. Condell, E. Asimakopoulou, and N. Bessis, "IoTs (Internet of Things) and DfPL (Device-free Passive Localisation) in a disaster management scenario," Simul. Model. Pract. Theory, vol. 35, pp. 86-96, Jun. 2013.

[7] M. Youssef, A. Agrawala, and A. Udaya Shankar, "WLAN location determination via clustering and probability distributions," in Proceedings of the First IEEE International Conference on Pervasive Computing and Communications, 2003. (PerCom 2003)., 2003, no. May 2014, pp. 143-150.

[8] M. Youssef, M. Mah, and A. Agrawala, "Challenges: Device-free Passive Localization for Wireless Environments," in Proceedings of the 13th annual ACM international conference on Mobile computing and networking - MobiCom '07, 2007, vol. 65, no. 5, p. 222.

[9] M. Seifeldin, A. Saeed, A. E. Kosba, A. El-Keyi, and M. Youssef, "Nuzzer: A Large-Scale Device-Free Passive Localization System for Wireless Environments," IEEE Trans. Mob. Comput., vol. 12, no. 7, pp. 1321-1334, Jul. 2013.

[10] K. Deepika and J. Usha, "Design \&amp; development of location identification using RFID with WiFi positioning systems," in 2017 Ninth International Conference on Ubiquitous and Future Networks (ICUFN), 2017, pp. 488-493.

[11] G. Deak, K. Curran, and J. Condell, "Evaluation of Smoothing Algorithms for a RSSI-Based Device-Free Passive Localisation," in Advances in Intelligent and Soft Computing, vol. 84, 2010, pp. 469-476.

[12] P. Bahl and V. N. Padmanabhan, "RADAR: an in-building RF-based user location and tracking system," in Proceedings IEEE INFOCOM 2000. Conference on Computer Communications. Nineteenth Annual Joint Conference of the IEEE Computer and Communications Societies (Cat. No.00CH37064), 2000, vol. 2, pp. 775-784.

[13] M. Youssef and A. Agrawala, "The Horus WLAN location determination system," in Proceedings of the 3rd 
international conference on Mobile systems, applications, and services - MobiSys '05, 2005, vol. 65, no. 7, p. 205.

[14] L. M. Ni, Yunhao Liu, Yiu Cho Lau, and A. P. Patil, "LANDMARC: indoor location sensing using active RFID," in Proceedings of the First IEEE International Conference on Pervasive Computing and Communications, 2003. (PerCom 2003)., 2003, pp. 407-415.

[15] H. Aly and M. Youssef, "New insights into wifi-based device-free localization," Proc. 2013 ACM Conf. Pervasive ubiquitous Comput. Adjun. Publ. - UbiComp '13 Adjun., pp. 541-548, 2013.

[16] A. E. Kosba, A. Saeed, and M. Youssef, "RASID: A Robust WLAN Device-free Passive Motion Detection System," 2012 IEEE Wirel. Commun. Netw. Conf., pp. 3284-3289, May 2011.

[17] A. Saeed, A. E. Kosba, and M. Youssef, "Ichnaea: A Low-Overhead Robust WLAN Device-Free Passive Localization System," IEEE J. Sel. Top. Signal Process., vol. 8, no. 1, pp. 5-15, Feb. 2014.

[18] J. Xiao, K. Wu, Y. Yi, L. Wang, and L. M. Ni, "Pilot: Passive Device-Free Indoor Localization Using Channel State Information," in 2013 IEEE 33rd International Conference on Distributed Computing Systems, 2013, pp. $236-245$.

[19] W. Yang et al., "Enhancing the Performance of Indoor Device-Free Passive Localization," Int. J. Distrib. Sens. Networks, vol. 11, no. 11, p. 256162, Nov. 2015.

[20] E. Vattapparamban, B. S. Ciftler, I. Guvenc, K. Akkaya, and A. Kadri, "Indoor occupancy tracking in smart buildings using passive sniffing of probe requests," in 2016 IEEE International Conference on Communications Workshops (ICC), 2016, pp. 38-44.

[21] F. Potortì, A. Crivello, M. Girolami, P. Barsocchi, and E. Traficante, "Localising crowds through Wi-Fi probes," Ad Hoc Networks, vol. 75-76, pp. 87-97, Jun. 2018.

[22] C. Xu, B. Firner, Y. Zhang, R. Howard, J. Li, and X. Lin, "Improving RF-based device-free passive localization in cluttered indoor environments through probabilistic classification methods," in 2012 ACM/IEEE 11th International Conference on Information Processing in Sensor Networks (IPSN), 2012, pp. 209-220.

[23] C. Xu et al., "SCPL: Indoor Device-Free Multi-Subject Counting and Localization Using Radio Signal Strength," in Proceedings of the 12th international conference on Information processing in sensor networks - IPSN '13, 2013, p. 79.

[24] C. Xu, M. Gao, B. Firner, Y. Zhang, R. Howard, and J. Li, "Towards robust device-free passive localization through automatic camera-assisted recalibration," in Proceedings of the 10th ACM Conference on Embedded Network Sensor Systems - SenSys '12, 2012, p. 339.

[25] J. Liu, N. An, M. T. Hassan, G. Chen, and Y. Zhang, "Poster Abstract: Node Deployment Mechanism for Quick, Indoor, and Device-Free Localization," in 2016 15th ACM/IEEE International Conference on Information Processing in Sensor Networks (IPSN), 2016, pp. 1-2.

[26] J. Liu, N. An, M. T. Hassan, M. Peng, Z. Cui, and S. Zhao, "Redundancy reduction for indoor device-free localization," Pers. Ubiquitous Comput., vol. 21, no. 1, pp. 5-15, Feb. 2017.

[27] D. Lieckfeldt, J. You, and D. Timmermann, "Passive Tracking of Transceiver-Free Users with RFID," in Communications in Computer and Information Science, vol. 53 CCIS, 2009, pp. 319-329.

[28] L. Yao, W. Ruan, Q. Z. Sheng, X. Li, and N. J. G. Falkner, "Exploring Tag-Free RFID-Based Passive Localization and Tracking via Learning-Based Probabilistic Approaches," in Proceedings of the 23rd ACM International Conference on Conference on Information and Knowledge Management - CIKM '14, 2014, pp. 1799-1802.

[29] W. Ruan, Q. Z. Sheng, L. Yao, X. Li, N. J. G. Falkner, and L. Yang, "Device-free human localization and tracking with UHF passive RFID tags: A data-driven approach," J. Netw. Comput. Appl., vol. 104, no. December 2016, pp. 78-96, Feb. 2018.

[30] B. Wagner, D. Timmermann, G. Ruscher, and T. Kirste, "Device-free user localization utilizing artificial neural networks and passive RFID," in 2012 Ubiquitous Positioning, Indoor Navigation, and Location Based Service (UPINLBS), 2012, pp. 1-7.

[31] L. Yao et al., "RF-Care: Device-Free Posture Recognition for Elderly People Using A Passive RFID Tag Array," in Proceedings of the 12th EAI International Conference on Mobile and Ubiquitous Systems: Computing, Networking and Services, 2015.

[32] J. Han et al., "Twins: Device-Free Object Tracking Using Passive Tags," IEEE/ACM Trans. Netw., vol. 24, no. 3, pp. 1605-1617, Jun. 2016.

[33] J. Wang, J. Xiong, H. Jiang, X. Chen, and D. Fang, "D-Watch: Embracing 'Bad' Multipaths for Device-Free Localization With COTS RFID Devices," IEEE/ACM Trans. Netw., vol. 25, no. 6, pp. 3559-3572, Dec. 2017.

[34] A. Popleteev, "Device-free indoor localization using ambient radio signals," in Proceedings of the 2013 ACM conference on Pervasive and ubiquitous computing adjunct publication - UbiComp '13 Adjunct, 2013, vol. 6, no. 1, pp. 549-552.

[35] A. Popleteev and T. Engel, "Device-Free Indoor Localization Based on Ambient FM Radio Signals," Int. J. Ambient Comput. Intell., vol. 6, no. 1, pp. 35-44, Jan. 2014.

[36] J. Wang, X. Feng, Q. Gao, X. Zhang, and M. Jin, "FM-based Device-Free Localization and Activity Recognition via Sparse Representation," in Proceedings of the 1st Workshop on Context Sensing and Activity Recognition - CSAR '15, 2015, pp. 7-12.

[37] Z. Tian, L. Shao, M. Zhou, and X. Wang, "A highly-accurate device-free passive motion detection system using cellular network," in 2016 IEEE Wireless Communications and Networking Conference, 2016, vol. 2016-Septe, no. Wenc, pp. 1-6.

[38] J. Hong and T. Ohtsuki, "Signal Eigenvector-Based Device-Free Passive Localization Using Array Sensor," IEEE Trans. Veh. Technol., vol. 64, no. 4, pp. 1354-1363, Apr. 2015.

[39] Y. Kilic, H. Wymeersch, A. Meijerink, M. J. Bentum, and W. G. Scanlon, “An experimental study of UWB device- 
free person detection and ranging," in 2013 IEEE International Conference on Ultra-Wideband (ICUWB), 2013, pp. $43-48$.

[40] C. Xu, "Device-free people counting and localization," in Proceedings of the 2013 ACM conference on Pervasive and ubiquitous computing adjunct publication - UbiComp '13 Adjunct, 2013, vol. 41, no. 4, pp. 367-372.

[41] J. Wilson and N. Patwari, "Radio Tomographic Imaging with Wireless Networks," IEEE Trans. Mob. Comput., vol. 9, no. 5, pp. 621-632, May 2010.

[42] J. S. C. Turner et al., "The study of human movement effect on Signal Strength for indoor WSN deployment," in 2013 IEEE Conference on Wireless Sensor (ICWISE), 2013, pp. 30-35.

[43] S. Shukri et al., "Analysis of RSSI-based DFL for human detection in indoor environment using IRIS mote," in 2016 3rd International Conference on Electronic Design (ICED), 2016, pp. 216-221.

[44] B. Wagner, N. Patwari, and D. Timmermann, "Passive RFID tomographic imaging for device-free user localization," in 2012 9th Workshop on Positioning, Navigation and Communication, 2012, no. 1, pp. 120-125.

[45] X. Zheng, J. Yang, Y. Chen, and H. Xiong, "An Adaptive Framework Coping with Dynamic Target Speed for Device-Free Passive Localization,” IEEE Trans. Mob. Comput., vol. 14, no. 6, pp. 1138-1150, Jun. 2015.

[46] M. Youssef and A. Agrawala, "Small-scale compensation for WLAN location determination systems," in 2003 IEEE Wireless Communications and Networking, 2003. WCNC 2003., 2003, vol. 3, no. Figure 1, pp. 1974-1978.

[47] A. Eleryan, M. Elsabagh, and M. Youssef, "Synthetic Generation of Radio Maps for Device-Free Passive Localization," in 2011 IEEE Global Telecommunications Conference - GLOBECOM 2011, 2011, pp. 1-5.

[48] M. Moussa and M. Youssef, "Smart cevices for smart environments: Device-free passive detection in real environments," in 2009 IEEE International Conference on Pervasive Computing and Communications, 2009, no. January, pp. 1-6.

[49] A. E. Kosba, A. Abdelkader, and M. Youssef, "Analysis of a Device-Free Passive Tracking System in Typical Wireless Environments," in 2009 3rd International Conference on New Technologies, Mobility and Security, 2009, no. January 2014, pp. 1-5.

[50] M. Seifeldin and M. Youssef, "A deterministic large-scale device-free passive localization system for wireless environments," in Proceedings of the 3rd International Conference on PErvasive Technologies Related to Assistive Environments - PETRA '10, 2010, p. 1.

[51] M. A. Seifeldin, A. F. El-keyi, and M. A. Youssef, "Kalman filter-based tracking of a device-free passive entity in wireless environments," in Proceedings of the 6th ACM international workshop on Wireless network testbeds, experimental evaluation and characterization - WiNTECH '11, 2011, p. 43.

[52] E. A. Wan, A. S. Paul, and P. G. Jacobs, "Tag-free RSSI based indoor localization," Inst. Navig. Int. Tech. Meet. 2012, ITM 2012, vol. 1, pp. 940-944, 2012.

[53] Z. Zhou, Z. Yang, C. Wu, L. Shangguan, and Y. Liu, "Omnidirectional Coverage for Device-Free Passive Human Detection,” IEEE Trans. Parallel Distrib. Syst., vol. 25, no. 7, pp. 1819-1829, Jul. 2014.

[54] F. Viani et al., "Passive wireless localization strategies for security in large indoor areas," in 2014 IEEE Conference on Antenna Measurements \& Applications (CAMA), 2014, pp. 1-3.

[55] K. Ohara, T. Maekawa, Y. Kishino, Y. Shirai, and F. Naya, "Transferring positioning model for device-free passive indoor localization," in Proceedings of the 2015 ACM International Joint Conference on Pervasive and Ubiquitous Computing - UbiComp '15, 2015, pp. 885-896.

[56] Z. Tian, X. Zhou, M. Zhou, S. Li, and L. Shao, "Indoor device-free passive localization for intrusion detection using multi-feature PNN," in 2015 10th International Conference on Communications and Networking in China (ChinaCom), 2015, pp. 272-277.

[57] X. Li, S. Li, D. Zhang, J. Xiong, Y. Wang, and H. Mei, "Dynamic-MUSIC: Accurate Device-Free Indoor Localization," in Proceedings of the 2016 ACM International Joint Conference on Pervasive and Ubiquitous Computing - UbiComp '16, 2016, vol. 9, no. 1, pp. 196-207.

[58] L. Gong, W. Yang, C. Xiang, D. Man, M. Yu, and Z. Yin, "WiSal: Ubiquitous WiFi-Based Device-Free Passive Subarea Localization without Intensive Site-Survey," in 2016 IEEE Trustcom/BigDataSE/ISPA, 2016, pp. 11291136.

[59] M. Alkandari, D. Basu, and S. F. Hasan, "A Wi-Fi based passive technique for speed estimation in indoor environments," in 2017 2nd Workshop on Recent Trends in Telecommunications Research (RTTR), 2017, pp. 1-3.

[60] R. Zhou, J. Chen, X. Lu, and J. Wu, "CSI fingerprinting with SVM regression to achieve device-free passive localization," in 2017 IEEE 18th International Symposium on A World of Wireless, Mobile and Multimedia Networks (WoWMoM), 2017, pp. 1-9.

[61] X. Chen, C. Ma, M. Allegue, and X. Liu, "Taming the inconsistency of Wi-Fi fingerprints for device-free passive indoor localization," in IEEE INFOCOM 2017 - IEEE Conference on Computer Communications, 2017, no. May, pp. 1-9.

[62] Baden-Württemberg, “ESP32's Wi-Fi Range Extended to $10 \mathrm{~km}$ with a Directional Antenna.” [Online]. Available: https://www.espressif.com/en/media_overview/news/esp32's-wi-fi-range-extended-10-km-directional-antenna. [Accessed: 10-Jan-2019].

[63] N. Azmi et al., "Design and Development of Multi-Transceiver Lorafi Board consisting LoRa and ESP8266-Wifi Communication Module," in IOP Conference Series: Materials Science and Engineering, 2018.

[64] R. Gunasagaran, L. M. Kamarudin, and A. Zakaria, "Embedded Device Free Passive (EDfP) System: Sensitivity of ESP32," in 2018 IEEE Student Conference on Research and Development (SCOReD), 2018, pp. 1-5. 